Table 1. SUMmary OF CONSTANTS FOR ${ }^{208} \mathrm{PbO}$

\begin{tabular}{ccccccccc}
\multicolumn{2}{c}{ State } & $T_{0}$ & $\omega_{e}$ & $x e \omega e$ & $B e$ & $10^{3} \alpha_{e}$ & $r_{e} \AA$. & Notes \\
$E$ & $0^{+}$ & 34680 & {$[440]$} & - & {$[0 \cdot 2382]$} & {$[1 \cdot 4]$} & $2 \cdot 18_{3}$ & 1 \\
$D$ & 1 & 30464 & $530 \cdot 5$ & $2 \cdot 92$ & $0 \cdot 2711$ & $3 \cdot 1$ & $2 \cdot 046$ & 2 \\
$C^{\prime}$ & 1 & 25720 & {$[475]$} & - & $0 \cdot 248$ & $1 \cdot 8$ & $2 \cdot 14$ & 2,3 \\
$C$ & $0^{+}$ & 24108 & {$[534]$} & {$[3 \cdot 9]$} & $0 \cdot 254$ & 2 & $2 \cdot 11$ & 4 \\
$B$ & 1 & 22531 & $493 \cdot 4$ & $2 \cdot 26$ & $0 \cdot 2646_{3}$ & $2 \cdot 6$ & $2 \cdot 070_{2}$ & 2 \\
$a$ & $? 2$ & & & & & \\
$A$ & $0^{+}$ & 20088 & $451 \cdot 7$ & $3 \cdot 33$ & $0 \cdot 2586_{9}$ & $1 \cdot 3_{8}$ & $2 \cdot 095_{3}$ & \\
$X$ & $0^{+}$ & 0 & $721 \cdot 8$ & $3 \cdot 70$ & $0 \cdot 3073_{3}$ & $2 \cdot 1_{2}$ & $1 \cdot 921_{6}$ &
\end{tabular}

(1) There are strong perturbations in state $E$, so that the constant for this state are uncertain. The values of $v^{\prime}$ for this system have been increased by 1 , following a study of the isotope effect.

(2) Perturbations in $R, P$ and $Q$ branches at about the same value f $J^{\prime}$ are observed in some of the levels of $B, C^{\prime}$ and $D$. The form of these suggests that they are caused by one or more $\Omega=2$ states.

(3) The system $C^{\prime}-X$ was observed by Howell (ref. 9 ) as a single $v^{\prime \prime}=0$ progression.

(4) The values of $v^{\prime}$ for the $C-X$ system have been increased by 2 , following a study of the isotope effect.

If these are all bound states, there are predicted three absorption systems $0^{+} \leftarrow X$ and five systems $1 \leftarrow X$. The $0^{-}$and 2 states would be identifiable in the absorption spectrum only through interactions with the $\Omega=1$ states. The number and the character of the excited states so far observed suggest that their interpretation as case- $c$ states may be the correct one. It is hoped that work, now in progress, of the numerous perturbations in these systems will help to elucidate this problem.

R. F. BARROW

J. L. Deutsch

D. N. Travis

Physical Chemistry Laboratory, University of Oxford.

${ }^{1}$ Mulliken, R. S., The Threshold of Space, edit. by Zelikoff (London, 1957).

Vago, E. E., and Barrow, R. F., Proc. Phys. Soc., 68, 538 (1946).

${ }^{3}$ Barrow, R. F., Deutsch, J. J., Lagerqvist, A., and Westerlund, B. (in the press).

${ }^{4}$ Lagerqvist, A., Nilheden, G., and Barrow, R. F., Proc. Phys. Soc. A, 65, 419 (1952).

${ }^{5}$ Nilheden, G., Ark. Fys., 10, 19 (1955).

${ }^{6}$ Barrow, R. F., and Rowlinson, H. C., Proc, Roy. Soc., A, 224, 37 (1954).

: Herzberg, G., Molecular Spectra and Molecular Structure. 1. Spectra of Diatomic Molecules (New York, 1950).

${ }^{\natural}$ Christy, A., and Bloomenthal, S., Phys. Rev., 35, 46 (1930),

'Howell, H. G., Proc. Roy. Soc., A, 153, 683 (1936).

\section{Kirkendall Effect in Gases}

AN experiment has been described ${ }^{1}$ in which the motion of fine particles of ammonium chloride smoke in a diffusing gas mixture was considered evidence for a Kirkendall effect in gases. Only a rough semiquantitative interpretation could be given at the time in terms of the usual phenomenological theory of the Kirkendall effect. Subsequent theoretical and experimental work ${ }^{2,3}$ on gaseous diffusion has shown that the phenomenological theory cannot be correct for gases, but that the classical kinetic theory of gases is capable of accounting for the phenomenon quantitatively. We wish to point out that it is now possible to give a quantitative interpretation to the previous measurements.

The experimental results may be summarized in terms of the usual intrinsic diffusion coefficients. The observed motion of the finest particles of the smoke corresponded to a difference of intrinsic diffusion coefficients of $\left(D_{2}-D_{1}\right) \simeq 0.63 \mathrm{~cm} .{ }^{2} / \mathrm{sec}$. for $1=\mathrm{CF}_{2} \mathrm{Cl}_{2}$ and $2=\mathrm{H}_{2}$ at $20^{\circ} \mathrm{C}$. and $1 \mathrm{~atm}$., in the absence of gravity. This value is probably an upper limit, since the necessary correction for the rate of sedimentation of the smoke particles under gravity appeared to be somewhat too large as it was made.
If the smoke particles are much larger in diameter than the mean free path of the gas molecules, they should drift with the bulk velocity (that is, the mass average velocity) of the diffusing gas mixture and thus act as true Kirkendall markers. In this case the difference of the intrinsic diffusion coefficients is given by equation (23) of reference 2 , and can be written as :

$$
D_{2}-D_{1}=\frac{m_{1}-m_{2}}{x_{1} m_{1}+x_{2} m_{2}} D_{12}
$$

where the $m$ 's are the molecular masses, the $x$ 's are mole fractions, and $D_{12}$ is the interdiffusion coefficient, which has been found ${ }^{3}$ to have a value of $0.38 \mathrm{~cm} .{ }^{2} / \mathrm{sec}$. Taking $x_{1}=x_{2}=1 / 2$ (at the diffusion boundary), we calculate from (1) that $\left(D_{2}-D_{1}\right)=0.73 \mathrm{~cm} .{ }^{2} / \mathrm{sec}$. This is larger than the experimentally observed upper limit of $0.63 \mathrm{~cm} .{ }^{2} / \mathrm{sec}$., so the smoke particles cannot be regarded as true Kirkendall markers.

If, however, the smoke particles are smaller in diameter than the mean free path, their behaviour is different, and the theory for such a case has been given by Waldmann. According to his equation (7.20), the particles move with a velocity corresponding to :

$$
D_{2}-D_{1}=\frac{m_{1}^{1 / 2}-m_{2}^{1 / 2}}{x_{1} m_{1}^{1 / 2}+x_{2} m_{2}^{1 / 2}} D_{12}
$$

from which we calculate $\left(D_{2}-D_{1}\right)=0.59 \mathrm{~cm} .{ }^{2} / \mathrm{sec}$. This is in good agreement with the observed upper limit. In equation (2) we have assumed that Waldmann's coefficients of diffuse scattering of the gas molecules from the smoke particles were equal to unity, which is in accord with experiment ${ }^{5}$. It is apparent that the finest smoke particles are probably smaller than the mean free path. This is physically reasonable, since the relevant mean free path in the experiments was of the order of $0 \cdot 1 \mu$.

Although the smoke particles were thus not true Kirkendall markers, they could have been used as such, since their velocity was proportional to the bulk velocity, although not equal to it. From equations (1) and $(2)$ the relation is :

$v=\left[\frac{m_{1}-m_{2}}{m_{1}^{1 / 2}-m_{2}^{1 / 2}}\right]\left[\frac{x_{1} m_{1}^{1 / 2}+x_{2} m_{2}^{1 / 2}}{x_{1} m_{1}+x_{2} m_{2}}\right] v($ smoke $)(3)$

or :

$v=\left[1+2\left(m_{1} m_{2}\right)^{1 / 2} /\left(m_{1}+m_{2}\right)\right] v($ smoke $)$,

$$
\text { if } x_{1}=x_{2}
$$

where $v$ is the mass average (bulk) velocity and $v$ (smoke) is the velocity of the smoke particles.

\section{E. A. Mason}

Institute for Molecular Physics, University of Maryland, College Park, Md.

\section{MILXER}

P. C. Carman

National Chemical Research

Laboratory,

South African Council for

Scientific and Industrial

Research,

Pretoria.

${ }^{1}$ Miller, L., and Carman, P. C., Nature, 186, 549 (1960),

${ }_{2}^{2}$ McCarty, K. P., and Mason, E. A., Phys. Fluids, 3, 908 (1960).

${ }^{3}$ Miller, L., and Carman, P. C., Trans. Farad. Soc. (to be published)

"Waldmann, L., Z. Naturf., 14, a, 589 (1959).

schmitt, K. H., Z. Naturf., 14, a, 870 (1959). 